\title{
Accelerated 4D phase contrast MRI in skeletal muscle contraction
}

\author{
Valentina Mazzoli'1,2,3 (D) | Lukas M. Gottwald ${ }^{1}$ ｜ Eva S. Peper ${ }^{1}$ | Martijn Froeling | \\ Bram F. Coolen ${ }^{5}$ | Nico Verdonschot ${ }^{3,6}$ | Andre M. Sprengers ${ }^{3,6}$ | Pim van Ooij ${ }^{1}$ | \\ Gustav J. Strijkers ${ }^{2,5}$ | Aart J. Nederveen ${ }^{1}$ \\ ${ }^{1}$ Department of Radiology, Academic Medical Center, Amsterdam, The Netherlands \\ ${ }^{2}$ Biomedical NMR, Department of Biomedical Engineering, Eindhoven University of Technology, Eindhoven, The Netherlands \\ ${ }^{3}$ Orthopaedic Research Lab, Radboud UMC, Nijmegen, The Netherlands \\ ${ }^{4}$ Department of Radiology, University Medical Center Utrecht, Utrecht, The Netherlands \\ ${ }^{5}$ Biomedical Engineering and Physics, Academic Medical Center, Amsterdam, The Netherlands \\ ${ }^{6}$ Laboratory for Biomechanical Engineering, University of Twente, Enschede, The Netherlands
}

\section{Correspondence}

Valentina Mazzoli, Department of

Radiology, Room Z0-178, Academic

Medical Center, Meibergdreef 9,

NL-1105 AZ Amsterdam, The

Netherlands.

Email: vmazzoli2@gmail.com
Purpose: 3D time-resolved (4D) phase contrast MRI can be used to study muscle contraction. However, 3D coverage with sufficient spatiotemporal resolution can only be achieved by interleaved acquisitions during many repetitions of the motion task, resulting in long scan times. The aim of this study was to develop a compressed sensing accelerated 4D phase contrast MRI technique for quantification of velocities and strain rate of the muscles in the lower leg during active plantarflexion/dorsiflexion.

Methods: Nine healthy volunteers were scanned during active dorsiflexion/plantarflexion task. For each volunteer, we acquired a reference scan, as well as 4 different accelerated scans ( $\mathrm{k}$-space undersampling factors: 3.14X, 4.09X, 4.89X, and 6.41X) obtained using Cartesian Poisson disk undersampling schemes. The data was reconstructed using a compressed sensing pipeline. For each scan, velocity and strain rate values were quantified in the gastrocnemius lateralis, gastrocnemius medialis, tibialis anterior, and soleus.

Results: No significant differences in velocity values were observed as a function acceleration factor in the investigated muscles. The strain rate calculation resulted in one positive $\left(\mathrm{s}_{+}\right)$and one negative $\left(\mathrm{s}_{-}\right)$eigenvalue, whereas the third eigenvalue $\left(\mathrm{s}_{3}\right)$ was consistently 0 for all the acquisitions. No significant differences were observed for the strain rate eigenvalues as a function of acceleration factor.

Conclusions: Data undersampling combined with compressed sensing reconstruction allowed obtainment of time-resolved phase contrast acquisitions with 3D coverage and quantitative information comparable to the reference scan. The 3D sensitivity of the method can help in understanding the connection between muscle architecture and muscle function in future studies.

\section{KE Y W O R D S}

compressed sensing, phase contrast, MRI, muscle contraction, skeletal muscles, strain rate 


\section{1 | INTRODUCTION}

Mathematical models of skeletal muscles have been shown to contribute to our understanding of the musculoskeletal system and to optimize surgical treatment. ${ }^{1-3}$ However, due to lack of subject-specific input information, these models often need to rely on considerable simplifications and assumptions. For instance, it is often assumed that muscles shorten uniformly along their longitudinal axis, whereas evidence has been provided for more complex contraction patterns and it was shown that the direction of shortening is not aligned with the muscle fiber direction. ${ }^{4,5}$ Furthermore, differences in deformation patterns were observed between deep and superficial muscles ${ }^{4}$ and between proximal and distal locations. ${ }^{5,6}$ Due to the highly complex pattern in muscle contraction and deformation, 3D visualization of subjectspecific contracting muscles would be extremely valuable.

The quantification of muscle deformation occurring during contraction is also a very valuable clinical tool to discriminate between healthy and diseased muscles. Deformation is typically quantified in terms of strain, which is a local property of a tissue that indicates the amount of shortening and lengthening of an object along a given direction; or in terms of strain rate, which is the rate at which the deformation occurs in time. Strain has the same direction as strain rate and can be calculated by integrating strain rate over time.

Phase contrast (PC) is an MRI-based technique widely used in the cardiovascular field to measure velocity of blood flowing inside arteries and veins. PC relies on the application of motion-sensitizing gradients, which result in phase accumulation of moving spins proportional to their velocities and to the magnitude of velocity-encoding magnetic field gradients, the so-called VENC. Gradients are usually applied in 3 orthogonal directions, allowing for evaluation of tissue velocities in a $3 \mathrm{D}$ fashion.

In the field of muscle MRI, PC sequences have been applied to quantify, among other things, changes in fascicle length, ${ }^{7}$ strain rates, ${ }^{5,8}$ and muscle inertial forces, ${ }^{9}$ as well as to gather insight into the complex muscle interaction in tongue movement. ${ }^{10} \mathrm{PC}$ in skeletal muscles can either be performed in a real-time approach or in a cine (triggered) fashion. Real-time PC has the advantage that measured velocities are not averaged over several motion cycles, thus reducing the need for exact repetition of the task and making the measurement more independent from muscle fatigue. ${ }^{11}$ However, real-time PC imaging only allows for imaging of a single slice with velocity encoded in 1 direction. Encoding of velocity over 3 directions requires interleaved/synchronized acquisition and thus repetition of the task.

Even when interleaved (cine) techniques are used, PC acquisition is usually performed in a single-slice fashion. In fact, 3D coverage with a spatiotemporal resolution of $\sim 3 \times 3 \times 5 \mathrm{~mm}^{3}$ and $160 \mathrm{~ms}$ can only be achieved by interleaved acquisitions during many repetitions of a motion task, resulting in long scan times ( $>10 \mathrm{~min})$. Many repetitions of the motion task can lead to muscle fatigue, which can result in poor reproducibility and can hamper applications in diseased or injured subjects. In general, PC in skeletal muscle requires low VENC due to the relatively low typical velocity of muscles as compared to flowing blood in veins and arteries. The use of low VENC requires the use of stronger velocity-encoding gradients, which increase the effect of eddy currents. Although strong eddy currents can introduce significant phase shading that can hinder the quantification of velocity values, the use of low VENC is usually required to achieve sufficient velocity-to-noise ratio in skeletal muscles. In order to mitigate this effect, several correction strategies have been proposed. ${ }^{12}$ An additional drawback related to the use of low VENC is the lengthening in TE and TR, which lead to even longer scan times. Therefore, PC in skeletal muscle would greatly benefit from acceleration techniques to reduce the total acquisition time, which would also allow decrease of muscle fatigue because a reduced number of motion repetitions would be required.

Therefore, the aim of this study was to develop a compressed sensing ${ }^{13}$ accelerated 4D PC MRI technique for quantifying velocities and strain rates of skeletal muscles in the lower leg during active foot plantar- and dorsiflexion in a clinically feasible scan time.

\section{2 | METHODS}

\section{1 | Subjects}

We collected images of the right lower leg of 9 healthy volunteers (4 males, 5 females) with no abnormalities in the lower leg. We received informed consent from all the subjects prior to the study, according to our institution's regulations.

\section{2 | Data acquisition}

MR imaging was performed with an Ingenia 3T MRI scanner (Philips Healthcare, Best, Netherlands) using a 16-channel torso and 8-channel posterior coil. Subjects were placed supine, feet first in the scanner. The scan protocol consisted of a high-resolution mDixon scan, performed with the leg at rest, and a series of 4D (3D time-resolved) PC scans during active muscle contraction. In order to minimize gross motion of the leg during dynamic data acquisitions, as well as displacement over different acquisitions, sand bags were placed on either side of the imaged leg. The scan parameters for the mDixon scan were: RF-spoiled fast field echo (FFE) with flip angle $10^{\circ}, \mathrm{TR}=10 \mathrm{~ms}, 3$ echoes with $\mathrm{TE}_{1}=1.96 \mathrm{~ms}$, $\Delta \mathrm{TE}=1.3 \mathrm{~ms}$, matrix size $=400 \times 160 \times 150 \mathrm{~mm}^{3}$, and voxel size $=1 \times 1 \times 1 \mathrm{~mm}^{3}$. After the anatomical mDixon 

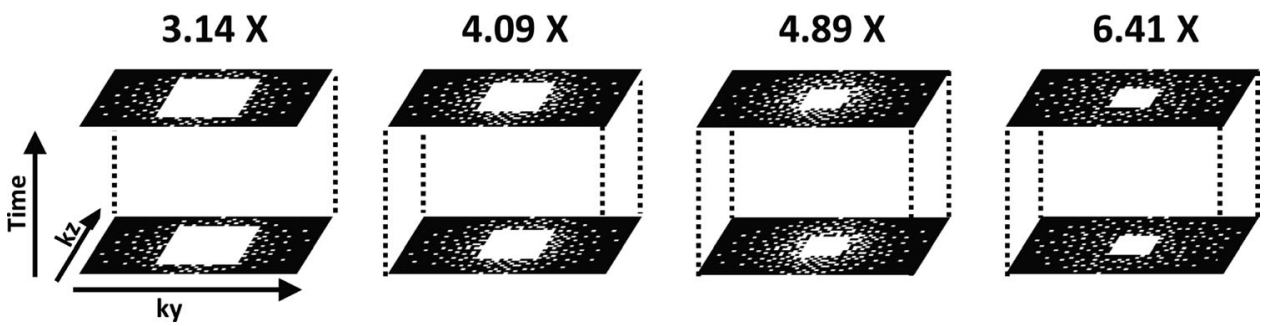

F I G URE 1 Undersampling patterns in (ky, kz)-space for the different acceleration factors (k-space undersampling factors: 3.14X, 4.09X, 4.89X, and $6.41 \mathrm{X})$. For all undersampling factors, the center of $\mathrm{k}$-space was fully sampled. The outer points followed a Poisson disk distribution with variable density

scan at rest, the volunteers were instructed to make continuous smooth movements with the right foot between approximately $30^{\circ}$ plantarflexion to $10^{\circ}$ dorsiflexion. In order to improve consistency of the range of motion, the maximum plantarflexed position was indicated to the subjects by using a triangular-shaped support under their feet. Each volunteer was instructed to start the motion in the maximum achievable plantarflexed position, move the foot toward a dorsiflexed position. and then return to the starting plantarflexed position. During the dynamic task, the volunteers received a visual cue from a bouncing ball displayed on a screen in the scanner room, with cycle duration of $2000 \mathrm{~ms}$ to guide their motion task. The MRI scan was motion-triggered at the same pace. Five time-resolved 3D turbo field echo (TFE) PC acquisitions were executed during the motion task: 1 reference scan and 4 accelerated acquisitions. To take into account the potential effect of muscle fatigue as a function of the scan number, the order in which the dynamic scans were performed was randomized. Scan parameters for the reference 3D TFE scan were: TFE factor $=3$, matrix size $=153 \times 53 \times 30$ (frequency encoding $\times$ phase encoding $\times$ slices), half scan in phase encoding direction $=0.6 \quad(32$ phase encoding lines), voxel size $=3 \times 3 \times 5 \mathrm{~mm}^{3}, \mathrm{TE} / \mathrm{TR}=4.5 / 6.4 \mathrm{~ms}$, $\mathrm{VENC}=12 \mathrm{~cm} / \mathrm{s}$ in 3 orthogonal directions (FH, AP, LR), and a temporal resolution of $160 \mathrm{~ms}$. This protocol resulted in a scan time of 32 (acquired phase encoding lines)/3 (TFE factor) $* 30$ (slices) $* 2 \mathrm{~s}=652 \mathrm{~s}$. The acquisition window within each TFE shot was $76.8 \mathrm{~ms}$, and a delay that depends on scanner-specific slew rate and duty cycle was introduced by the scanner software to accommodate gradient duty cycle. Accelerated acquisitions were performed with the same parameters, but without half scan and with different k-space undersampling schemes (k-space undersampling factors: 3.14X, 4.09X, 4.89X, and 6.41X), as shown in Figure 1. For the undersampled scans, the scanner software was modified to acquire a predefined number of sampling profiles. The sampling profiles $\left(\mathrm{k}_{\mathrm{y}}, \mathrm{k}_{\mathrm{z}}\right)$ were generated in MatLab (MathWorks Inc., Natick, MA) using the Berkeley Advanced Reconstruction Toolbox (BART),${ }^{14}$ and were designed according to a Poisson disk pattern with variable density. ${ }^{15}$ Total acquisition times for the 5 scans were 10:52 (reference scan), 5:38, 4:20, 3:38, and 2:46 min, which corresponded to $326,169,130,109$, and 83 executions of the motion task, respectively. All of the scans (mDixon, reference scan, and accelerated scans) were performed in the sagittal plane.

\section{3 | Image reconstruction}

Raw data was imported in MatLab (MathWorks) using ReconFrame (Gyrotools, Zurich, Switzerland), and compressed sensing reconstruction was performed using the BART, with a wavelet transform in space $(x, y, z)$ and an additional sparsifying total variation transform in space $(x, y, z)$ and time. The L1 -regularization was performed with a regularization parameter of $r=0.01$ for both wavelet and total variation transform, and 50 iteration steps.

\subsection{Data processing}

Four muscle groups in the lower leg (tibialis anterior [TA], soleus [SOL], gastrocnemius lateralis [GCL], and gastrocnemius medialis $[\mathrm{GCM}]$ ) were manually segmented on the water mDixon images. Segmentation was performed using ITKsnap. ${ }^{16}$ To account for the change in shape of the different muscle groups during the motion task, image registration was performed. For this purpose, the mDixon scan was downsampled to the same resolution of the PC data and registered to each motion phase of the magnitude scan. The registration pipeline was repeated for each of the 5 acquired datasets (reference scan and 4 accelerated scans), which consisted of a b-spline registration approach with 2 levels of resolution. All the registration steps were performed using Elastix. ${ }^{17}$ Subsequently, the transformation used to register the downsampled high-resolution scan was applied to the masks of each muscle group. This resulted in a set of muscle segmentations for each of the 12 time points. After image registration, the masks were eroded to exclude the voxels at the interface between adjacent muscle groups. These masks were subsequently used to extract the values of velocity and strain rate over the whole muscle groups.

\subsection{Strain rate calculation}

As a first preprocessing step, the PC data as well as the previously registered masks were resampled to isotropic 
resolution $\left(2 \times 2 \times 2 \mathrm{~mm}^{3}\right)$. Subsequently, the phase images were corrected by subtracting from each time frame the average phase over all the 12 time frames. This is based on the assumption that the net velocity over the full dorsiflexion/ plantarflexion cycle is $0 .{ }^{12}$ Because strain calculation involves the spatial gradients, which are extremely sensitive to noise, each 3D velocity image was filtered prior to further calculation. A 3D Savitzky-Golay filter ${ }^{18}$ was used for this purpose due to its generally good performance in denoising data while keeping peak values preserved.

Calculation of strain rate started from the measured 3D velocity vector $\mathrm{v}(\mathrm{x}, \mathrm{y}, \mathrm{z})=\mathrm{v}_{\mathrm{x}}(\mathrm{x}, \mathrm{y}, \mathrm{z}) \mathrm{x}+\mathrm{v}_{\mathrm{y}}(\mathrm{x}, \mathrm{y}, \mathrm{z}) \mathrm{y}+\mathrm{v}_{\mathrm{z}}(\mathrm{x}, \mathrm{y}, \mathrm{z})$ $\mathrm{z}$ and the $3 \mathrm{D}$ spatial gradient of this velocity vector:

$$
\boldsymbol{L}=\left(\begin{array}{lll}
\frac{\partial v_{x}}{\partial x} & \frac{\partial v_{x}}{\partial y} & \frac{\partial v_{x}}{\partial z} \\
\frac{\partial v_{y}}{\partial x} & \frac{\partial v_{y}}{\partial y} & \frac{\partial v_{y}}{\partial z} \\
\frac{\partial v_{z}}{\partial x} & \frac{\partial v_{z}}{\partial y} & \frac{\partial v_{z}}{\partial z}
\end{array}\right)
$$

The symmetric part of the strain rate tensor was then calculated according to:

$$
\boldsymbol{S}=\frac{1}{2}\left(\boldsymbol{L}+\boldsymbol{L}^{T}\right)=\frac{1}{2}\left(\begin{array}{ccc}
2 \frac{\partial v_{x}}{\partial x} & \frac{\partial v_{x}}{\partial y}+\frac{\partial v_{y}}{\partial x} & \frac{\partial v_{x}}{\partial z}+\frac{\partial v_{z}}{\partial x} \\
\frac{\partial v_{x}}{\partial y}+\frac{\partial v_{y}}{\partial x} & 2 \frac{\partial v_{y}}{\partial y} & \frac{\partial v_{y}}{\partial z}+\frac{\partial v_{z}}{\partial y} \\
\frac{\partial v_{x}}{\partial z}+\frac{\partial v_{z}}{\partial x} & \frac{\partial v_{y}}{\partial z}+\frac{\partial v_{z}}{\partial y} & 2 \frac{\partial v_{z}}{\partial z}
\end{array}\right) .
$$

The tensor $S$ was subsequently diagonalized to obtain the 3 eigenvalues. The eigenvalues were then sorted on a pixelby-pixel basis in the following order: maximum negative eigenvalue $\left(\mathrm{s}_{-}\right)$, maximum positive eigenvalue $\left(\mathrm{s}_{+}\right)$, and remaining eigenvalue $\left(s_{3}\right)$. The principal direction of strain can be considered in first approximation to be aligned with the axis of the muscles fibers within a given voxel. Therefore, the strain will be associated with negative eigenvalues $\left(\mathrm{s}_{-}\right)$during muscle contraction and positive values $\left(\mathrm{s}_{+}\right)$during muscle relaxation.

\section{6 | Statistical analysis}

One-way analysis of variance (ANOVA) was used to compare the mean FH velocities, the mean magnitude velocities, and the mean strain rates as a function of acceleration factors for the different muscle groups (GCL, GCM, SOL, and TA) for frame \#3 and frame \#9 (peak negative and peak positive velocity). Statistical analysis was performed in SPSS $^{19}$ (IBM Corp., Armonk, NY). A value of $P<0.05$ was considered to be significant.

\section{7 | Repeatability assessment}

In order to assess the repeatability of strain rate determination, a subset of volunteers $(n=5)$ was scanned on a second day. The protocol consisted of the reference scan and the scan with the highest acceleration factor (6.41X). After the 2 scans were completed, the subjects were allowed to rest for at least 5 minutes, without being repositioned, and then both scans were repeated. The agreement between the first and second eigenvalues of the strain rate tensor, calculated from the 2 different scans (reference and 6.41X), was evaluated with Bland-Altman analysis. For both eigenvalues, the 2 peak frames were considered (frame \#3 and frame \#9). The coefficient of variation (CV) for strain rate was calculated by dividing the SD of the paired difference by the mean of all measurements.

\section{3 | RESULTS}

The data acquisition was successful for all volunteers. All results were visually inspected and judged to be of sufficient quality to continue with further analysis. Figures 2 and 3 show velocity images reconstructed from undersampled data in comparison to the reference scan for a male volunteer. Phase images clearly show consistency with the muscle function. In fact, antagonist muscles (posterior and anterior compartments) show opposite velocity in both visualized frames (\#3 and \#9). The average velocities for the 4 muscle groups are shown in Figure 4 (mean \pm SD).

The mean peak velocities averaged over all 9 subjects, together with their SDs, are shown in Table 1A (FH velocity) and Table 1B (magnitude velocity) for the 4 investigated muscle groups (GCL, GCM, SOL, and TA).

No significant differences were observed for the velocity values in the 2 peak frames (\#3 and \#9) as function of undersampling factor for either FH velocity or velocity magnitude. Smaller peak velocity values were observed in the soleus muscle as compared to the other muscle groups.

The strain rate tensor was calculated for all volunteers. For all subjects, the first 2 principal eigenvalues of the strain tensor were one high positive $\left(\mathrm{s}_{+}\right)$and one high negative $\left(s_{-}\right)$value. The third eigenvalue $\left(s_{3}\right)$ was near 0 for all investigated muscle groups and for all volunteers. Figures 5 and 6 show the complex geometrical pattern of the principal positive and negative strain rate eigenvector across the gastrocnemius muscle group and posterior aponeurosis. In Figure 5, the principal strain rate eigenvector in the gastrocnemius during plantarflexion is oriented in a similar way to the known fiber direction (as indicated by the anatomy drawing), indicating that during active muscle activation the principal direction of contraction was roughly oriented along the fiber direction, whereas local 


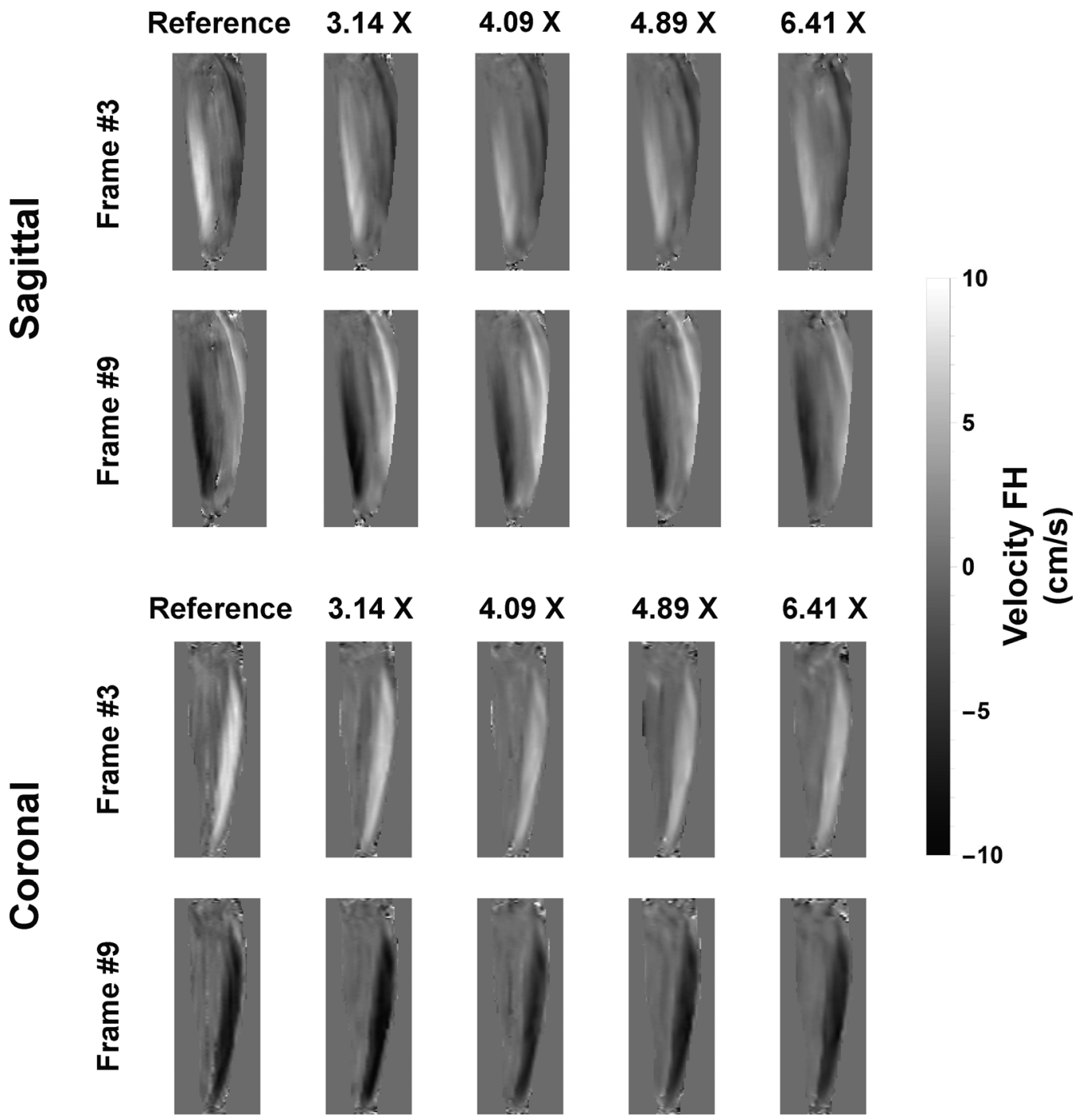

FIG URE 2 Representative FH velocity for a volunteer in the sagittal and coronal planes for the reference scan and for the 4 accelerated scans. The antagonist function of the muscles in the posterior and anterior compartment can be observed for all accelerated scans, as indicated by the opposite sign of the velocity

FH, feet-head.

expansion occurred along the cross-sectional area of the fibers. In frame \#3, representing the dorsiflexion phase, the principal direction of contraction was oriented perpendicular to the principal eigenvector observed during contraction, indicating fascicle axial elongation and compression along the perpendicular direction. The same behavior is observed in Figure 6 for the posterior compartment, with muscle shortening during contraction in the plantarflexion task (frame \#9) and muscle elongation during dorsiflexion (frame \#3).

The 3 peak eigenvalues $\left(s_{-}, s_{+}\right.$, and $\left.s_{3}\right)$ of the strain rate tensor, averaged over all the volunteers, are visualized in Figure 7. These values were calculated on the whole muscle volumes. No significant differences were observed in the 3 eigenvalues as a function of the acceleration factor for any of the investigated muscles $(P>0.8)$. The sum of the 3 eigenvalues over the full muscle volume was nearly 0 for all volunteers. The third eigenvalue was not significantly different from 0 for any muscle or acceleration factor. The time evolution of the 3 eigenvalues is shown in the supporting information material (Supporting Information Figure S1: GCL, Supporting Information Figure S2: GCM, Supporting Information Figure S3: SOL, Supporting Information Figure S4: TA).

The Bland-Altman plots for the first and second eigenvalues of the strain rate tensor are shown in Figure 8. The scan/rescan experiment showed no bias for both the reference and the 6.41X scan. For all investigated muscles, the SD of the scan/rescan differences was smaller for the accelerated scan as compared to the reference scan, as shown in the Bland-Altman plots. The CV for the 4 different muscles (GCL, GCM, SOL, and TA) for the reference and the 6.41X scan is reported in Table 2 . The $\mathrm{CV}$ is generally smaller for the accelerated scan, as compared to the reference scan, 


\section{Frame \#3 Frame \#9}

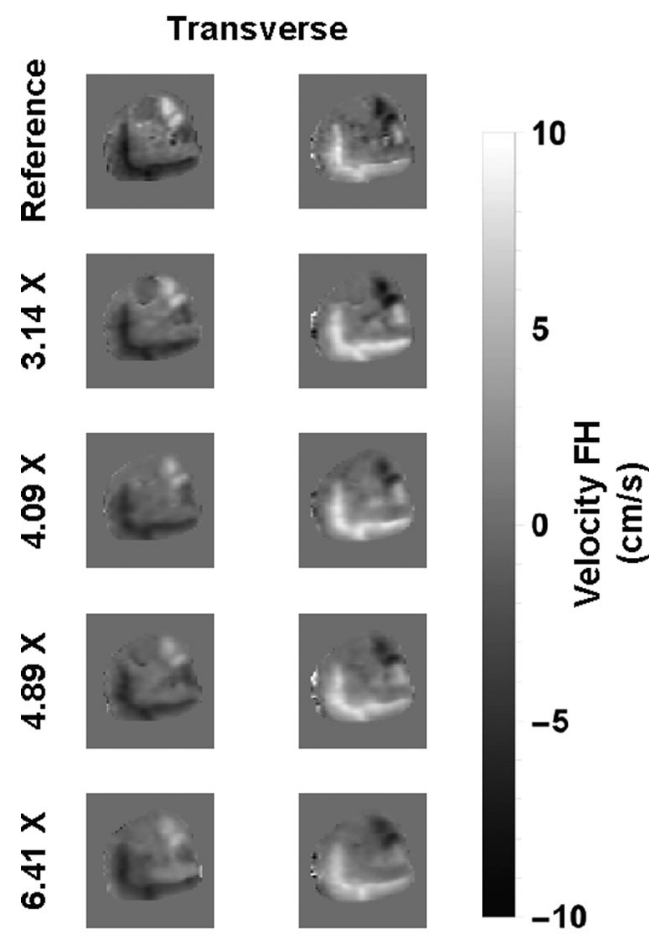

FIG URE 3 Representative FH velocity for a volunteer in the transverse plane for the reference scan and for the 4 accelerated scans. The antagonist function of the muscles in the posterior and anterior compartment can be observed for all accelerated scans, as indicated by the opposite sign of the velocity

indicating improved repeatability. Smaller CV in the reference scan are observed only for the second eigenvalue of SOL and the first eigenvalue of TA.

\section{4 | DISCUSSION}

The purpose of this study was to apply 4D PC MRI to characterize muscle velocity and strain rate in the full lower leg during active foot dorsiflexion/plantarflexion and to explore the feasibility of accelerating the data acquisition by using data undersampling and compressed sensing reconstruction.

The comparison of undersampled 4D PC-MRI data to the data obtained with the reference scan showed no significant differences in velocity values, even up to a $\mathrm{k}$-space undersampling factor of 6.41X. Although deviations in the velocity curves in each single frame may exist, on average, reference and highly undersampled data provide the same results for velocity and strain rate, thus facilitating a significant decrease in scan time from 10:52 min for the reference scan down to $2: 46 \mathrm{~min}$ with the highest $\mathrm{k}$-space undersampling.

For some of the volunteers, for whom a very high level of consistency could be achieved during the execution of the motion task, the peak velocity values in the lower leg showed a small reduction as a function of acceleration, which is related to the known temporal smoothing involved in compressed sensing reconstruction. ${ }^{20}$ However, significant temporal smoothing and consequent reduction of peak velocity values also are to be expected in case of problems with repeating the motion task consistently over time. This effect could be especially dramatic in case of long scan times and is expected to decrease with a decreasing number of motion repetitions needed. Consequently, the effects of small reduction in the peak velocity values are compensated by the increased likelihood of successful repetition of the motion task, as indicated by the fact that no significant differences were observed in mean velocity as a function of increasing acceleration factor. Our data show that, with the use of compressed sensing, we could recover highly undersampled data, thus highly reducing the scan duration and number of repetitions of the task that the subjects have to perform. The achieved decrease in the number of repetitions needed can be beneficial for healthy subjects because it can decrease discomfort and fatigue and is of great importance for patients, who will likely present a reduced ability to consistently perform a repetitive task. PC-MRI patient scans are warranted to further improve in the future by real-time approaches.

Our repeatability study compared the performance of the reference scan and of the scan with the highest acceleration factor in the assessment of peak strain rate values. Although the number of subjects scanned is small $(n=5)$, and more subjects would be needed to systematically compare the two acquisitions, our data suggest a higher consistency in the repetition of the motion task for shorter scan times. Furthermore, the CV obtained indicate a generally better performance of the highly accelerated scan with respect to the reference scan. The general better repeatability of the accelerated scans highlights the importance of short scan time for reliable quantification of strain rate in skeletal muscles. In particular, the shorter scan time achieved in this study can be useful to reduce muscle fatigue and can be of particular benefit to patients who have less tolerance to repetitive motion and long scan times. The CV obtained in this study could be further decreased in future studies by using a properly designed device during the execution of the motion task, which could further improve consistency.

Our experimental setup does not allow for the definition of gold standard measurements due to the possible variability in the execution of the motion task within a single subject and the quality of the repeatability depending on the scan time. Furthermore, our reference scan was not a fully sampled scan but used half-scan encoding. This was done because a fully sampled scan would have required an excessively long scan time ( $>15$ minutes). Pilot experiments for the purpose of protocol optimization showed that it was impossible for the volunteers to perform the motion task consistently during such a long scan time. 


\section{Gastrocnemius Lateralis}
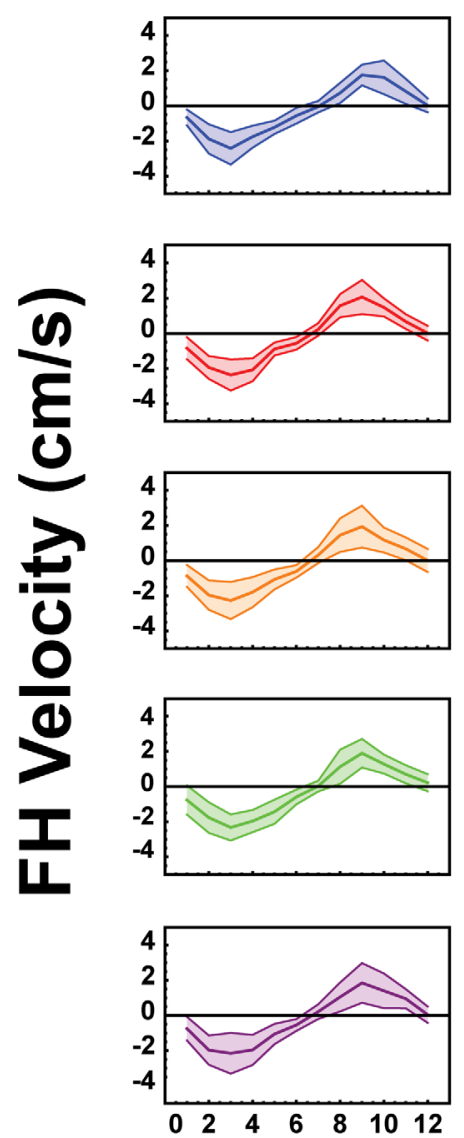

Gastrocnemius Medialis
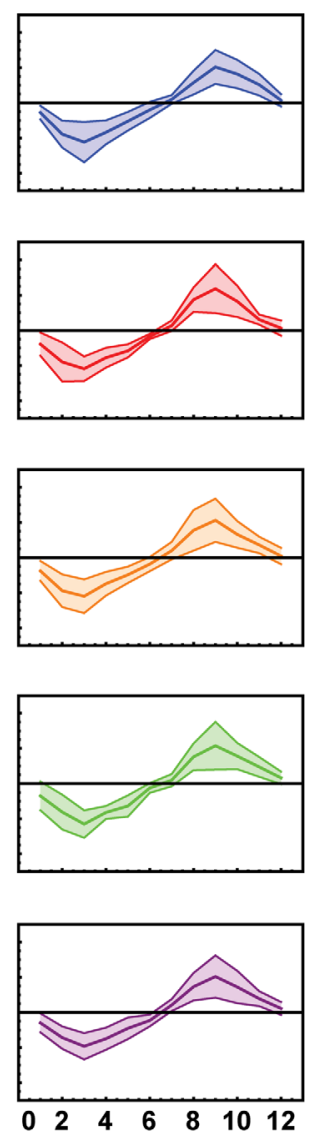

Soleus
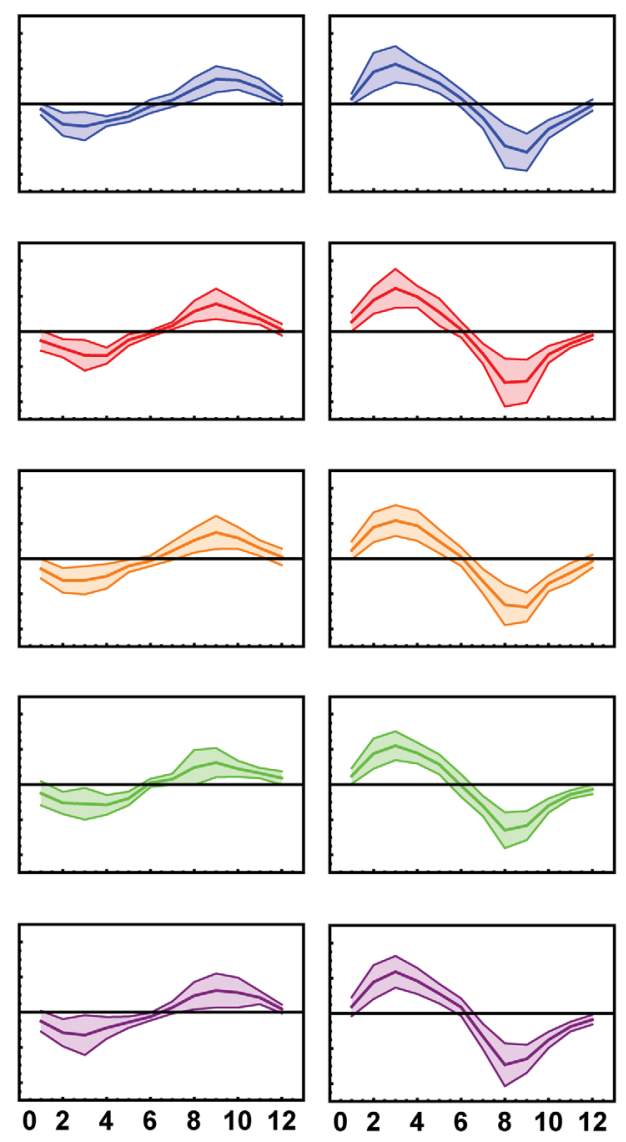

Frame \#
Reference

$3.14 X$

$4.09 X$

$4.89 X$

$6.41 X$

F I G URE 4 FH velocity averaged over all subjects (mean = thick line, $\mathrm{SD}=$ shaded area). No significant differences were observed as a function of k-space undersampling factor (blue: reference scan, red: 3.14X, orange: 4.09X, green: 4.89X, and purple: 6.41X). All results are presented for 4 different muscles in the lower leg (GCL, GCM, SOL, and TA). The opposite signs of the muscles in the posterior compartment and the TA are consistent with the muscles working in opposite directions

GCL, gastrocnemius lateralis; GCM, gastrocnemius medialis; SOL, soleus; TA, tibialis anterior.

The peak velocity values in our study were in agreement with previously published results performed under similar low-load conditions. ${ }^{9,12,21}$ Furthermore, the velocity data obtained were suitable for calculation of strain rate for all the volunteers. The 3D strain tensor showed consistent patterns across volunteers. In particular, the first 2 eigenvalues were always one big positive and one big negative value, whereas the third eigenvalue was consistently nearly 0 . This is consistent with the presence of one shortening and one lengthening direction of muscle fibers, and the Poisson ratio of skeletal muscle being close to 0.5 . This finding of near incompressibility of skeletal muscle tissue is in agreement with previous studies. ${ }^{419,22,23}$

Detailed information on muscle contraction can be obtained using ultrasonography techniques ${ }^{22-24}$ with high temporal and spatial resolution. However, these measurements usually provide a small FOV. Aside from ultrasounds, other MRI techniques, including MR tagging and displacement encoding with stimulated echoes (DENSE), are available that allow in vivo measurements of strain characteristics with a large FOV. In MR tagging, a prepulse applied prior to image readout creates a spatial modulation of the magnetization with a periodic function. This results in a line or in a grid of low signal intensity superimposed on the anatomical images. The tag lines can be manually or automatically tracked, and directly provide information on tissue displacement. Following the distortion of these tag lines over time allows for measurement of soft tissue deformation. MR tagging techniques have been used to monitor tissue displacement during active isometric contraction in humans, ${ }^{12}$ as well as during muscle indentation both in rats ${ }^{25}$ and in healthy humans. ${ }^{26,27}$ The main drawbacks of MR tagging are that it is commonly a $2 \mathrm{D}$ technique with limited spatial resolution, and that tag lines fade due to $\mathrm{T}_{1}$ relaxation, which limits the duration of the motion cycle that can be assessed. Furthermore, MR tagging often requires manual or 
TABLE 1 (A) FH velocities and (B) velocity magnitudes in the GCL, GCM, SOL, and TA, averaged over the muscle groups and over the 9 volunteers

\begin{tabular}{|c|c|c|c|c|c|c|c|c|}
\hline & \multicolumn{2}{|l|}{ GCL } & \multicolumn{2}{|l|}{ GCM } & \multicolumn{2}{|l|}{ SOL } & \multicolumn{2}{|l|}{ TA } \\
\hline \multicolumn{9}{|c|}{ A. Velocity FH (cm/s) } \\
\hline Frame \# & 3 & 9 & 3 & 9 & 3 & 9 & 3 & 9 \\
\hline Ref. scan & $-2.42(0.92)$ & $1.75(0.59)$ & $-2.23(1.15)$ & $2.04(0.96)$ & $-1.27(0.80)$ & $1.40(0.74)$ & 2.25 & $-2.75(1.06)$ \\
\hline $3.14 X$ & $-2.37(0.89)$ & $2.07(0.97)$ & $-2.18(0.70)$ & $2.37(1.38)$ & $-1.36(0.87)$ & $1.56(0.87)$ & $2.45(1.11)$ & $-2.83(1.22)$ \\
\hline $4.09 \mathrm{X}$ & $-2.27(1.06)$ & $1.93(1.19)$ & $-2.20(1.00)$ & $2.12(1.23)$ & $-1.23(0.80)$ & $1.49(0.94)$ & $2.17(0.87)$ & $-2.80(0.82)$ \\
\hline $4.89 \mathrm{X}$ & $-2.33(0.74)$ & $1.90(0.81)$ & $-2.30(0.78)$ & $2.15(1.36)$ & $-1.09(0.90)$ & $1.24(0.83)$ & $2.21(0.82)$ & $-2.33(0.82)$ \\
\hline $6.41 \mathrm{X}$ & $-2.15(1.16)$ & $1.85(1.13)$ & $-1.92(0.74)$ & $2.04(1.20)$ & $-1.30(1.13)$ & $1.24(1.00)$ & $2.37(0.90)$ & $-2.58(0.80)$ \\
\hline \multicolumn{9}{|c|}{ B. Magnitude Velocity $(\mathrm{cm} / \mathrm{s})$} \\
\hline Frame \# & 3 & 9 & 3 & 9 & 3 & 9 & 3 & 9 \\
\hline Ref. scan & $2.91(1.00)$ & $2.81(0.80)$ & $2.69(1.14)$ & $2.68(0.68)$ & $2.06(0.81)$ & $2.20(0.71)$ & 2.55 & $3.00(1.07)$ \\
\hline $3.14 X$ & $2.84(0.91)$ & $2.96(1.04)$ & $2.63(0.73)$ & $2.90(1.36)$ & $2.16(0.80)$ & $2.15(0.87)$ & $2.72(1.02)$ & $3.10(1.24)$ \\
\hline $4.09 \mathrm{X}$ & $2.75(1.00)$ & $2.77(1.13)$ & $2.61(0.93)$ & $2.67(1.11)$ & $1.98(0.74)$ & $2.06(0.91)$ & $2.50(0.76)$ & $2.97(0.86)$ \\
\hline $4.89 \mathrm{X}$ & $2.90(0.79)$ & $2.75(1.13)$ & $2.74(0.72)$ & $2.64(1.31)$ & $2.09(0.63)$ & $1.78(0.68)$ & $2.57(0.70)$ & $2.43(0.84)$ \\
\hline $6.41 \mathrm{X}$ & $2.94(0.83)$ & $2.68(0.97)$ & $2.45(0.72)$ & $2.52(1.15)$ & $2.16(0.82)$ & $1.90(0.79)$ & $2.75(0.81)$ & $2.67(0.78)$ \\
\hline
\end{tabular}

Velocities $( \pm \mathrm{SD}$ ) are presented for 2 time frames (frame \#3 and frame \#9). No significant differences in velocity were observed as a function of the k-space undersampling factor $(P>0.05)$.

FH, feet-head; GCL, gastrocnemius lateralis; GCM, gastrocnemius medialis; ref., reference; SOL, soleus; TA, tibialis anterior.

semiautomated tracking of tag lines, which is a laborious and time-consuming task.

To decrease user interaction for quantification of displacement and strain in MR tagging data, techniques such as harmonic phase (HARP) have been proposed, ${ }^{28,29}$ for which strain can be directly computed from the local spatial frequency of tag lines. Although this approach does not require the tracking of tag lines and may facilitate strain analysis, it still does not provide advantages over MR tagging in terms of spatial resolution of strain.

On the other hand, PC MRI approaches allow for the acquisition of velocity information encoded in each pixel of

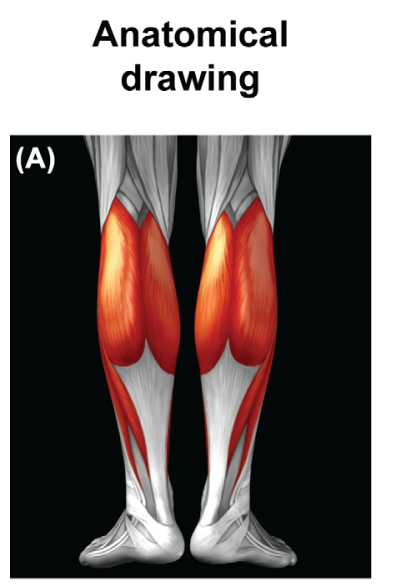

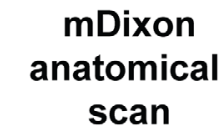

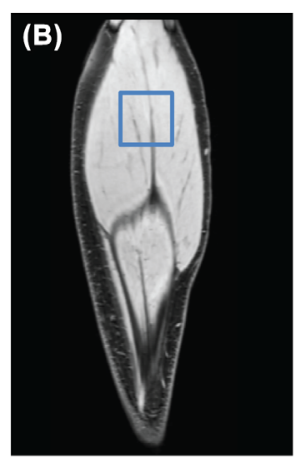

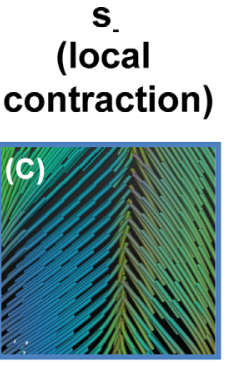

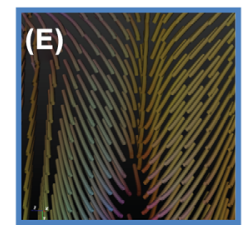

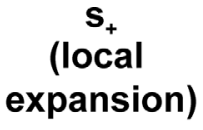

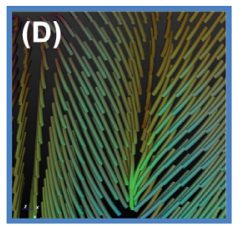

\section{Frame \#3 (Dorsiflexion)}

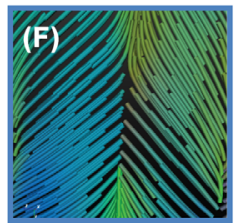

\section{Frame \#9} (Plantarflexion)

F I G URE 5 Color-coded strain rate images in a ROI in the gastrocnemius muscle for one volunteer. (a) Anatomical drawing of the lower leg. (b) mDixon anatomical scan. (c-f) Color-coded strain rate images obtained in the ROI indicated in b). This dataset was acquired with a k-space undersampling factor of 6.41X. The strain rate is color-coded according to the standard convention: green: FH, red: AP, and blue: LR. The tracts represent the principal direction of contraction $\left(\mathrm{s}_{-}\right)$(c and $\left.\mathrm{e}\right)$ and the principal direction of expansion $\left(\mathrm{s}_{+}\right)(\mathrm{d}$ and $\mathrm{f})$

FH, feet-head; AP, anterior-posterior; LR, left-right; ROI, region of interest. 


\section{Anatomical drawing}

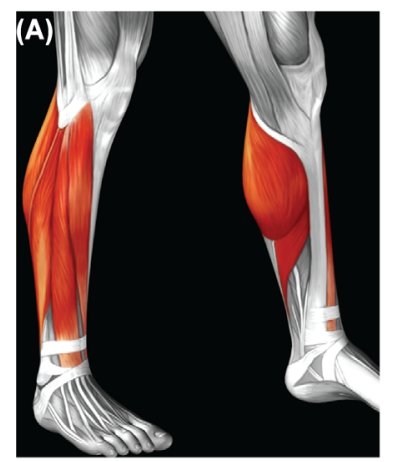

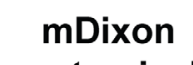
anatomical scan

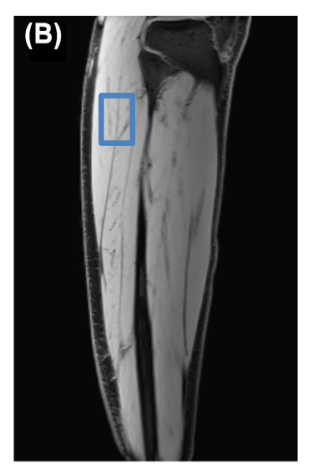

S. (local contraction)
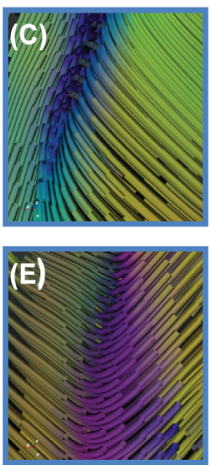

$\mathbf{s}_{+}$ (local
expansion)

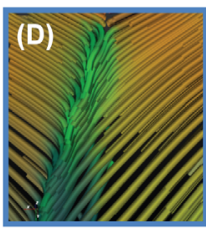

\section{Frame \#3 (Dorsiflexion)}

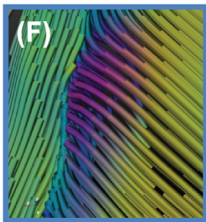

Frame \#9 (Plantarflexion)

F I G URE 6 Color-coded strain rate images in a ROI in the posterior aponeurosis for one volunteer. (a) Anatomical drawing of the lower leg. (b) mDixon anatomical scan. (c-f) Color-coded strain rate images obtained in the ROI indicated in (b). This dataset was acquired with a k-space undersampling factor of 6.41X. The strain rate is color-coded according to the standard convention: green: FH, red: AP, blue: LR. The tracts represent the principal direction of contraction $\left(\mathrm{s}_{-}\right)$(c and e) and the principal direction of expansion $\left(\mathrm{s}_{+}\right)(\mathrm{d}$ and $\mathrm{f})$

the image, thereby providing intrinsically higher resolution as compared to MR tagging and HARP. Another MR-based displacement encoding technique that can provide strain values on a pixel-by-pixel basis is DENSE. ${ }^{30}$ Although DENSE is conceptually similar to PC because it encodes displacement on the phase of the signal, it is based on the acquisition of stimulated echoes rather than primary echoes. DENSE has been successfully applied to the study of muscle contraction $^{31}$ and cartilage deformation under cyclic loading. ${ }^{32-34}$ One of the advantages of DENSE is that it allows for encoding displacements over long time intervals, which is especially beneficial for the study of skeletal muscles contraction, characterized by low velocities. However, the use of a stimulated echo approach leads to a reduction in SNR by a factor 2 , which is usually compensated for by decreasing the spatial resolution or by increasing the number of acquisitions, ${ }^{35}$ thus leading to much longer scan time.

A great advantage of our method compared to previous work is the fully $3 \mathrm{D}$ nature of the acquisition. To the best of our knowledge, this is the first study to measure muscle deformation in the entire lower leg with a full 4D sensitivity (full 3D volume with velocity encoding over 3 orthogonal directions), which enables a full 3D analysis of strain rate and velocity. This is especially important because many muscles present a curved geometry, which may be difficult to correctly depict using a single plane. Our 3D results show the presence of a strain rate component close to 0 in the cross section of the fibers, indicating a planar pattern of strain development in agreement with findings by Englund et al. ${ }^{4}$ This planar pattern would imply that a $2 \mathrm{D}$ measurement would suffice, provided that the MR scan can be precisely planned along the directions of the fascicles, as shown in previous studies focusing on the gastrocnemius medialis. ${ }^{36}$ However, precise identification of fascicle orientation based on anatomical scans can be challenging, and some muscle groups present a more complex geometry, which does not allow for a definition of a suitable constant plane parallel to the axis of the fibers. ${ }^{37}$ Therefore, with a $3 \mathrm{D}$ approach, as presented in this study, a strain rate information can be derived even for muscles with complex geometry and curvature, without relying on precise geometry planning based on the direction of muscle fascicles. In addition to difficulties in geometry planning for $2 \mathrm{D}$ imaging, another challenge is posed by muscle groups that undergo rotation during motion, which may be erroneously interpreted as contraction when imaging only in a single plane. Furthermore, the ability to image a 3D volume implies that no assumptions were needed on the planar nature of the motion or on local volume conservation $^{38}$ as these parameters could be measured directly from the data. Another advantage of our acquisition scheme is that it allows for a pixel-by-pixel-based analysis instead of the more common ROI-based analysis. The ability to perform a pixel-by-pixel analysis allows for the $3 \mathrm{D}$ visualization of the principal strain rate directions, as demonstrated in this work. Although a visual inspection of the strain rate maps revealed a nonhomogeneous pattern between the distal and proximal portions of the muscles, as well as differences between the superficial and deep regions-as already highlighted by previous studies ${ }^{4,5}$ - we report only the average value per muscle group. An in-depth analysis of these regional differences was outside the scope of this work.

Previous studies have correlated the anatomical fiber direction with the principal direction of strain. Englund et al. ${ }^{4}$ measured the TA muscle during isometric dorsiflexion contraction using MR tagging and combined these data with fiber direction obtained from DT-MRI for the same subjects. They found an average difference of $40^{\circ}$ between the principal direction of strain and the fiber direction in the superficial 
Frame \#3
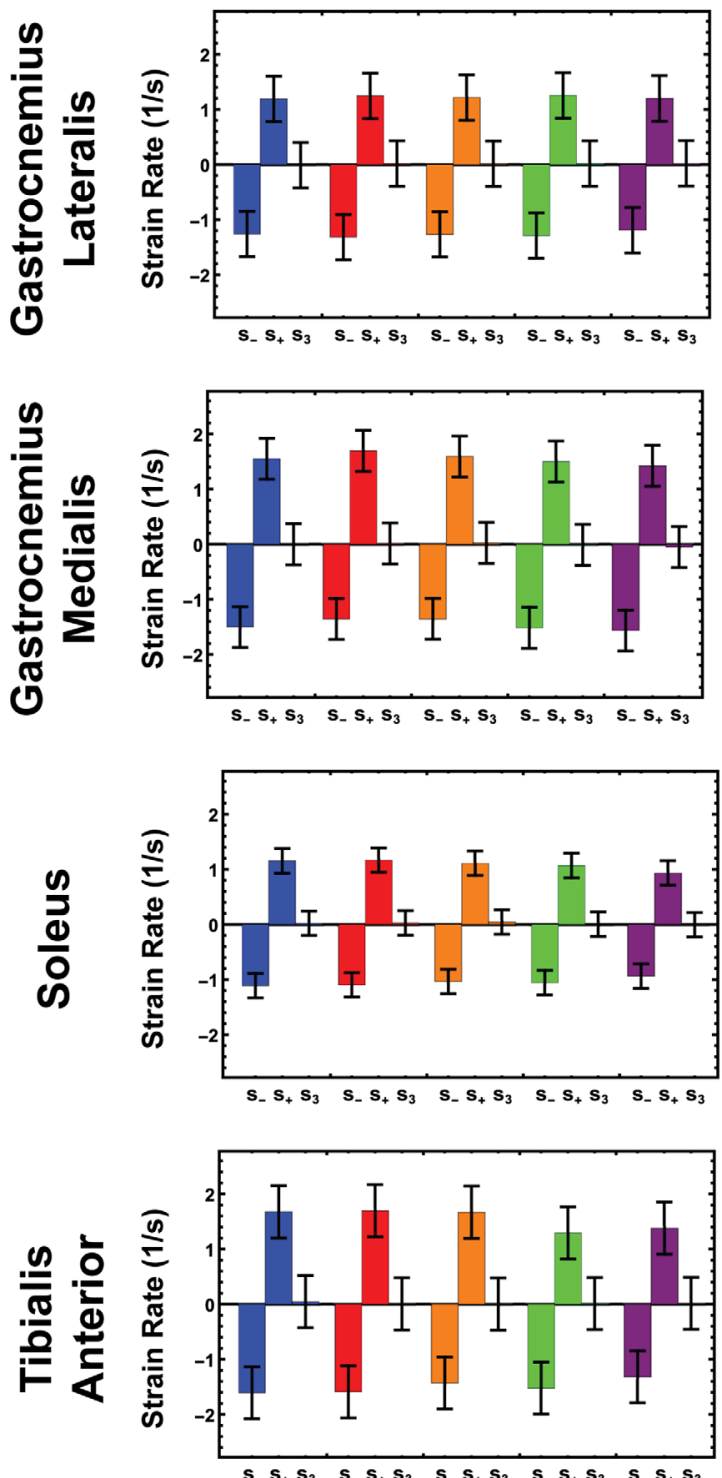

Frame \#9

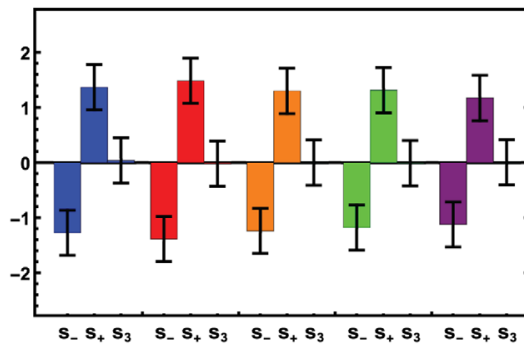

Reference

$3.14 \mathrm{X}$

$4.09 \mathrm{X}$

$4.89 \mathrm{X}$

$\square .41 \mathrm{X}$
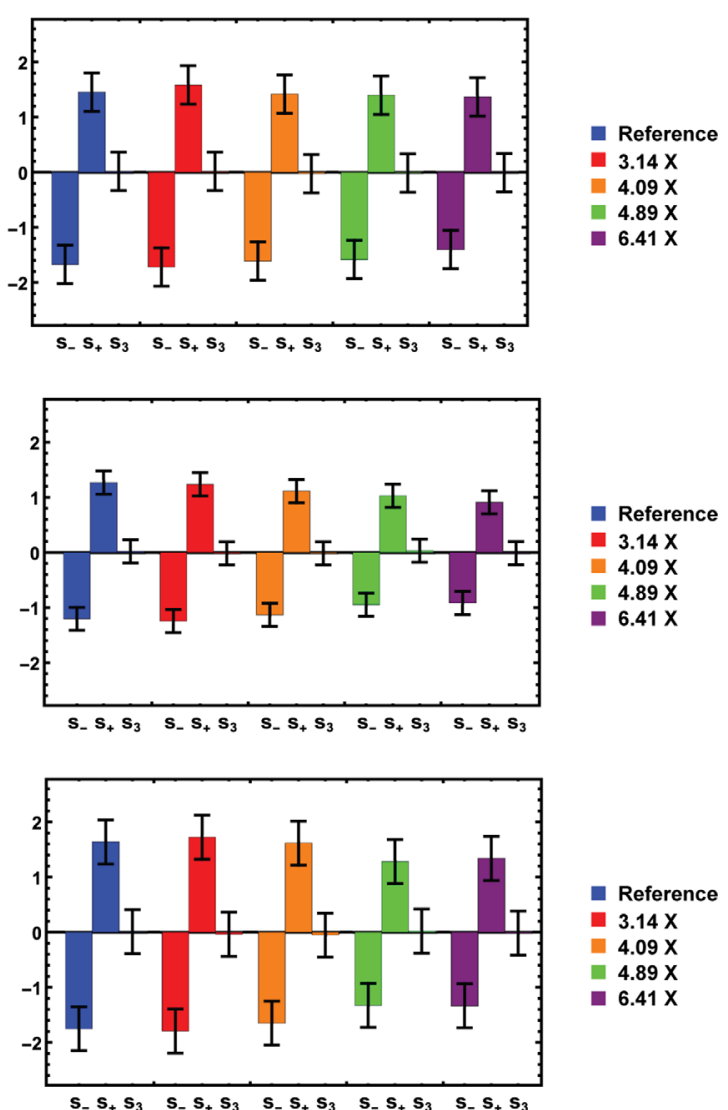

F I G U RE 7 Strain rate eigenvalues $\left(\mathrm{s}_{-}, \mathrm{s}_{+}\right.$, and $\mathrm{s}_{3}$ ) calculated over the whole muscle groups (GCL, GCM, SOL, and TA). The results are averaged over all the subjects, and error bars indicate SDs. Third principal strain rate was not significantly different from 0 . No significant differences were observed as a function of the $\mathrm{k}$-space undersampling factor (blue: reference scan, red: 3.14X, orange: 4.09X, green: 4.89X and purple: 6.41X)

compartment, and a difference of $20^{\circ}$ in the deep compartment. At first, this shows a complex and nonuniform pattern of contraction, probably due to fibers with different lengths and different connective tissue arrangements across the muscle. Similar findings were reported by Sinha et al. ${ }^{5}$ and Malis et al., ${ }^{8}$ who observed a location-dependent difference in fiber angle (approximately $45^{\circ}$ for the proximal gastrocnemius and approximately $25^{\circ}$ for the distal gastrocnemius). However, these previously reported data were based on small ROI analysis. Due to the 3D nature of the acquisition in our study, our data can potentially be combined with DT-MRI measurements at a local level, which could help elucidate the complex geometrical arrangement of muscle fiber strain and its connection with the underlying anatomy.
This study has a few limitations. First of all, due to the very long reference acquisition we used, which required a high number of repetitions of the motion task, we were limited to low-load conditions. However, considering that we can obtain consistent patterns for velocity and strain rate even with high undersampling factors, higher loads could be investigated in the future using our method. An additional limitation of our scan method is that the measurements are still partially dependent on the ability of the subjects to perform the dorsiflexion/plantarflexion task in a very repeatable way. While reducing the number of repetitions needed increases the likelihood of successful motion repetition, the dependency on the subject's motion skills is not completely eliminated. Furthermore, in our study we did not measure the force 


\section{Gastrocnemius Lateralis}

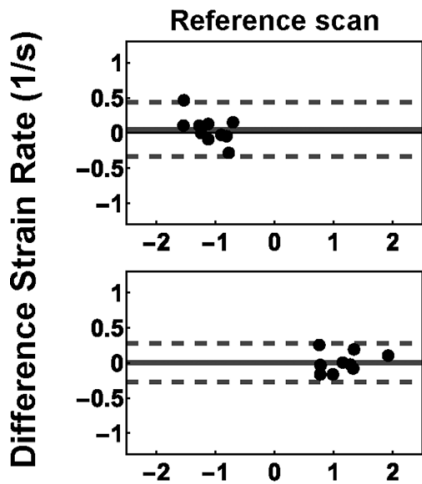

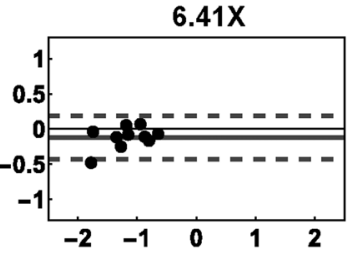

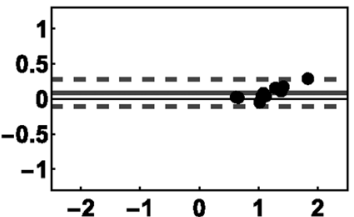

Soleus

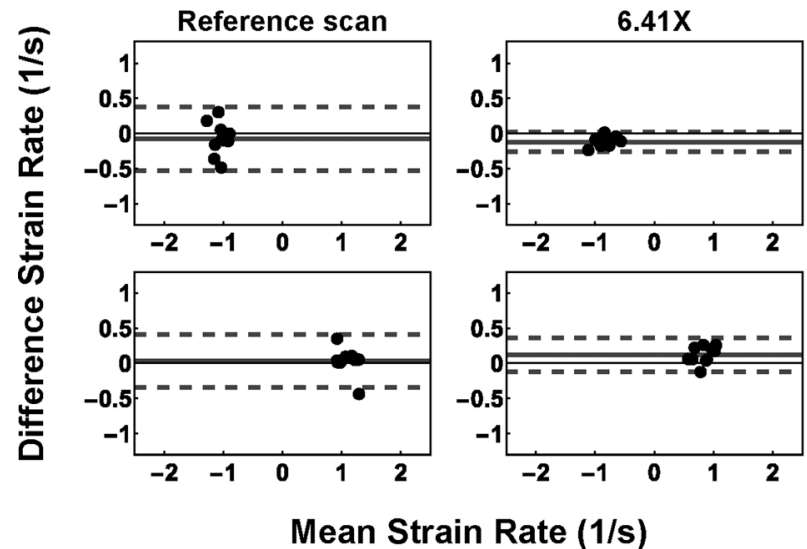

Gastrocnemius Medialis
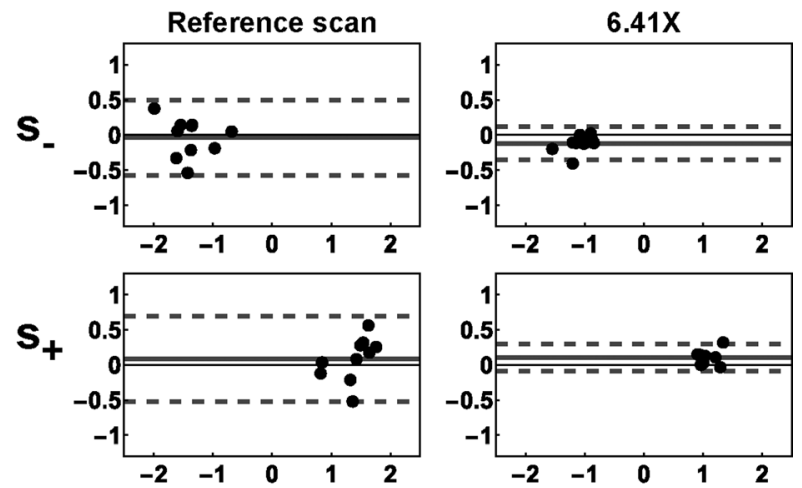

Tibialis Anterior
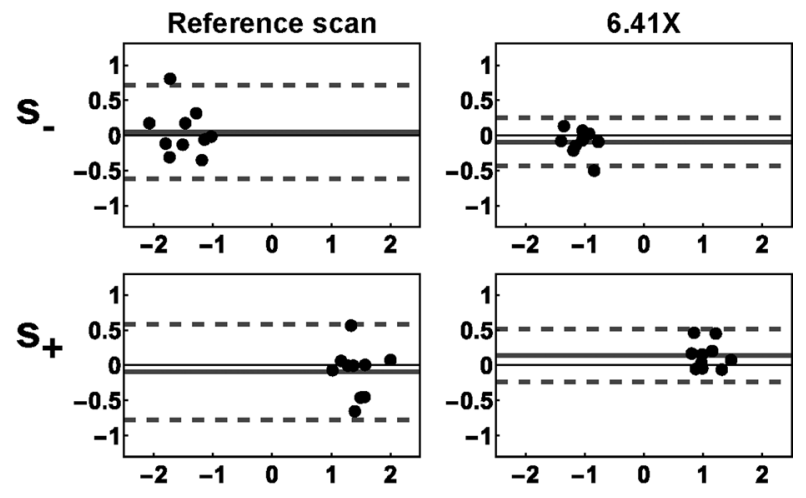

Mean Strain Rate (1/s)

F I G URE 8 Bland-Altman plots for peak values of the 1st and 2nd eigenvalue of the strain rate tensor for 4 muscles (GCL, GCM, SOL, and TA) and different acceleration factors (reference scan and 6.41X). The gray lines indicate the mean value of scan-rescan measurements (solid) and $1.96 \mathrm{SD}$ of the paired differences (dashed)

exerted by the subjects, and we could not ensure that the same percentage maximum voluntary contraction was maintained. While that the consistency of percentage maximum voluntary contraction effort maintenance is desirable in physiological experiments, since the main aim of this study was the development of an accelerated PC-MRI protocol using compressed sensing, these were not carried out in the project. Consistent execution of the motion task with constant percentage

TABLE 2 CV for strain rate values determined from scan/rescan of reference scan and $6.41 \mathrm{X}$ accelerated scan. The CVs for 1 st and 2nd eigenvalues of strain rate tensor are reported for 4 muscle groups (GCL, GCM, SOL, and TA)

\begin{tabular}{|c|c|c|c|c|c|c|c|c|}
\hline & GCL & & GCM & & SOL & & TA & \\
\hline \multicolumn{9}{|c|}{ Strain Rate (1/s) } \\
\hline & $1 \mathrm{st}$ & 2 nd & $1 \mathrm{st}$ & 2 nd & $1 \mathrm{st}$ & 2nd & $1 \mathrm{st}$ & 2nd \\
\hline Ref. scan & 12.40 & 10.43 & 12.60 & 14.63 & 15.30 & 11.93 & 14.78 & 17.13 \\
\hline $6.41 X$ & 10.09 & 6.01 & 9.85 & 8.81 & 11.51 & 13.98 & 14.92 & 16.49 \\
\hline
\end{tabular}

$\mathrm{CV}$, coefficient of variation; GCL, gastrocnemius lateralis; GCM, gastrocnemius medialis; ref., reference; SOL, soleus; TA, tibialis anterior. maximum voluntary contraction is even more challenging for a subject to maintain; therefore, our proposed method could greatly benefit these experiments as well.

Advances in MRI acceleration techniques may further decrease the scan time for the acquisition of a full dataset in the future. In our study, we used a constant undersampling scheme over time. However, other undersampling strategies exist that can create unique sampling patterns for each time frame, which in turn allow for higher acceleration factors by exploiting additional sparsifying transforms over time. ${ }^{39}$ Therefore, time-varying undersampling patterns may improve the results in future studies and allow for higher acceleration factors than those presented in this study. Ideally, a real-time $\mathrm{PC}$ acquisition is required for subjects who find it difficult to consistently comply with the motion task. However, real-time approaches are currently limited to single-slice acquisition with a single velocity-encoded direction. ${ }^{11}$

\section{5 | CONCLUSION}

In conclusion, this study demonstrated that it is possible to reconstruct highly undersampled 4D PC MRI data of the 
muscles in the lower leg during active plantar- and dorsiflexion using a compressed sensing acquisition and reconstruction scheme. This strategy facilitates a significant decrease in scan time while keeping comparable velocity values. Furthermore, the data was suitable for strain rate calculation. Further research will focus on combining motion with structural data and applying these methods in subjects with muscle pathologies, such as sarcopenic subjects. These results, coupled to accurate structural information, may lead to a deeper understanding of movement abnormalities and optimization of treatment/intervention strategies.

\section{ACKNOWLEDGMENT}

We would like to thank Philips Healthcare, in particular Maarten Versluis, for help in creating the prospective undersampling software patch. A compiled version of the patch is available upon request (a.j.nederveen@amc.uva.nl).

\section{ORCID}

Valentina Mazzoli (D) http://orcid.org/0000-0002-6700-8424

\section{REFERENCES}

[1] Blemker SS, Pinsky PM, Delp SL. A 3D model of muscle reveals the causes of nonuniform strains in the biceps brachii. J Biomech. 2005;38:657-665.

[2] Blemker SS, Asakawa DS, Gold GE, Delp SL. Image-based musculoskeletal modeling: applications, advances, and future opportunities. J Magn Reson Imaging. 2007;25:441-451.

[3] Asakawa DS, Blemker SS, Gold GE, Delp SL, Asakawa DS. In vivo motion of the rectus femoris muscle after tendon transfer surgery. J Biomech. 2002;35:1029-1037.

[4] Englund EK, Elder CP, Xu Q, Ding Z, Damon BM. Combined diffusion and strain tensor MRI reveals a heterogeneous, planar pattern of strain development during isometric muscle contraction. Am J Physiol Regul Integr Comp Physiol. 2011;300:R1079-R1090.

[5] Sinha U, Malis V, Csapo R, Moghadasi A, Kinugasa R, Sinha S. Age-related differences in strain rate tensor of the medial gastrocnemius muscle during passive plantarflexion and active isometric contraction using velocity encoded MR imaging: potential index of lateral force transmission. Magn Reson Med. 2015;73:1852-1863.

[6] Finni T, Hodgson JA, Lai AM, Edgerton VR, Sinha S. Nonuniform strain of human soleus aponeurosis-tendon complex during submaximal voluntary contractions in vivo. J Appl Physiol. 2003;95:829-837.

[7] Shin DD, Hodgson JA, Edgerton VR, Sinha S. In vivo intramuscular fascicle-aponeuroses dynamics of the human medial gastrocnemius during plantarflexion and dorsiflexion of the foot. J Appl Physiol. 2009;107:1276-1284.

[8] Malis V, Sinha U, Csapo R, Narici M, Sinha S. Relationship of changes in strain rate indices estimated from velocity-encoded MR imaging to loss of muscle force following disuse atrophy. Magn Reson Med. 2018;79:912-922.
[9] Wentland AL, McWalter EJ, Pal S, Delp SL, Gold GE. Muscle velocity and inertial force from phase contrast MRI. J Magn Reson Imaging. 2015;42:526-532.

[10] Felton SM, Gaige TA, Reese TG, Wedeen VJ, Gilbert RJ. Mechanical basis for lingual deformation during the propulsive phase of swallowing as determined by phase-contrast magnetic resonance imaging. J Appl Physiol. 2007;103:255-265.

[11] Asakawa DS, Nayak KS, Blemker SS, et al. Real-time imaging of skeletal muscle velocity. J Magn Reson Imaging. 2003;18: 734-739.

[12] Sinha S, Hodgson JA, Finni T, Lai AM, Grinstead J, Edgerton VR. Muscle kinematics during isometric contraction: development of phase contrast and spin tag techniques to study healthy and atrophied muscles. J Magn Reson Imaging. 2004;20:1008-1019.

[13] Lustig M, Donoho D, Pauly JM. Sparse MRI: the application of compressed sensing for rapid MR imaging. Magn Reson Med. 2007;58:1182-1195.

[14] Uecker M, Ong F, Tamir JI, et al. Berkeley Advanced Reconstruction Toolbox. In Proceedings of the 23rd Annual Meeting of ISMRM, Toronto, Canada, 2015. p. 2486.

[15] Hollingsworth KG. Reducing acquisition time in clinical MRI by data undersampling and compressed sensing reconstruction. Phys Med Biol. 2015;60:R297-R322.

[16] Yushkevich PA, Piven J, Hazlett HC, et al. User-guided 3D active contour segmentation of anatomical structures: significantly improved efficiency and reliability. Neuroimage. 2006;31: 1116-1128.

[17] Klein S, Staring M, Murphy K, Viergever MA, Pluim JPW. Elastix: a toolbox for intensity-based medical image registration. IEEE Trans Med Imaging. 2010;29:196-205.

[18] Savitzky A, Golay MJE. Smoothing and differentiation of data by simplified least squares procedures. Anal Chem. 1964;36: 1627-1639.

[19] Takaza M, Moerman KM, Gindre J, Lyons G, Simms CK. The anisotropic mechanical behaviour of passive skeletal muscle tissue subjected to large tensile strain. J Mech Behav Biomed Mater. 2012;17:209-220.

[20] Kim D, Dyvorne HA, Otazo R, Feng L, Sodickson DK, Lee VS. Accelerated phase-contrast cine MRI using k-t SPARSE-SENSE. Magn Reson Med. 2012;67:1054-1064.

[21] Sinha S, Shin DD, Hodgson JA, Kinugasa R, Edgerton VR. Computer-controlled, MR-compatible foot-pedal device to study dynamics of the muscle tendon complex under isometric, concentric, and eccentric contractions. J Magn Reson Imaging. 2012;36:498-504.

[22] Lopata RGP, van Dijk JP, Pillen S, et al. Dynamic imaging of skeletal muscle contraction in three orthogonal directions. $J$ Appl Physiol. 2010;109:906-915.

[23] Gijsbertse K, Sprengers AMJ, Nillesen MM, et al. Three-dimensional ultrasound strain imaging of skeletal muscles. Phys Med Biol. 2017;62:596-611.

[24] Sikdar S, Wei Q, Cortes N. Dynamic ultrasound imaging applications to quantify musculoskeletal function. Exerc Sport Sci Rev. 2014;42:126-135.

[25] Ceelen KK, Stekelenburg A, Mulders JLJ, et al. Validation of a numerical model of skeletal muscle compression with MR 
tagging: a contribution to pressure ulcer research. $J$ Biomech Eng. 2008;130:61015.

[26] Moerman KM, Sprengers AMJ, Simms CK, Lamerichs RM, Stoker J, Nederveen AJ. Validation of SPAMM tagged MRI based measurement of 3D soft tissue deformation. Med Phys. 2011;38:1248-1260.

[27] Moerman KM, Sprengers AMJ, Simms CK, Lamerichs RM, Stoker J, Nederveen AJ. Validation of continuously tagged MRI for the measurement of dynamic 3D skeletal muscle tissue deformation. Med Phys 2012;39:1793-810.

[28] Venkatesh BA, Gupta H, Lloyd SG, Dell'Italia L, Denney TS. 3D left ventricular strain from unwrapped harmonic phase measurements. J Magn Reson Imaging. 2010;31:854-862.

[29] Kuijer JPA, Hofman MBM, Zwanenburg JJM, Marcus JT, Van Rossum AC, Heethaar RM. DENSE and HARP: two views on the same technique of phase-based strain imaging. J Magn Reson Imaging. 2006;24:1432-1438.

[30] Aletras AH, Ding S, Balaban RS, Wen H. DENSE: displacement encoding with stimulated echoes in cardiac functional MRI. J Magn Reson. 1999;137:247-252.

[31] Zhong X, Epstein FH, Spottiswoode BS, Helm PA, Blemker SS. Imaging two-dimensional displacements and strains in skeletal muscle during joint motion by cine DENSE MR. J Biomech. 2008;41:532-540.

[32] Neu CP, Hull ML, Walton JH. Error optimization of a threedimensional magnetic resonance imaging tagging-based cartilage deformation technique. Magn Reson Med. 2005;54:1290-1294.

[33] Chan DD, Neu CP, Hull ML. In situ deformation of cartilage in cyclically loaded tibiofemoral joints by displacement-encoded MRI. Osteoarthr Cartil. 2009;17:1461-1468.

[34] Chan DD, Neu CP. Transient and microscale deformations and strains measured under exogenous loading by noninvasive magnetic resonance. PLoS One. 2012;7:e33463.

[35] Epstein FH, Gilson WD. Displacement-encoded cardiac MRI using cosine and sine modulation to eliminate (CANSEL) artifact-generating echoes. Magn Reson Med. 2004;52:774-781.

[36] Kinugasa R, Hodgson JA, Edgerton VR, Sinha S. Asymmetric deformation of contracting human gastrocnemius muscle. J Appl Physiol. 2012;112:463-70.

[37] Rana M, Hamarneh G, Wakeling JM. 3D curvatures of muscle fascicles in triceps surae. $J$ Appl Physiol. 2014;117:1388-1397.

[38] Sinha U, Csapo R, Malis V, Xue Y, Sinha S. Age-related differences in diffusion tensor indices and fiber architecture in the medial and lateral gastrocnemius. J Magn Reson Imaging. 2015;41:941-953.

[39] Hutter J, Grimm R, Forman C, Greiser A, Hornegger J, Schmitt P. Multi-dimensional flow-adapted compressed sensing (MDFCS) for time-resolved velocity-encoded Phase Contrast MRA. In Proceedings of the International Symposium on Biomedical Imaging, San Francisco, CA, USA, 2013. p. 13-16.

\section{SUPPORTING INFORMATION}

Additional supporting information may be found in the online version of this article.

FIGURE S1 Time evolution of the three strain rate eigenvalues $\left(s_{-}, s_{+}\right.$, and $s_{3}$ ) for the Gastrocnemius Lateralis muscle (GCL). The results are averaged over all the subjects (mean, thick line and SD, thin lines). No significant differences are observed as a function of the acceleration factor (blue: reference scan, red: 3.14X, orange: 4.09X, green: $4.89 \mathrm{X}$ and purple: $6.41 \mathrm{X}$ ) for the peak strain rates during contraction (frame \#9) and during relaxation (frame \#3).

FIGURE S2 time evolution of the three strain rate eigenvalues $\left(s_{-}, s_{+}\right.$, and $\left.s_{3}\right)$ for the Gastrocnemius Medialis muscle (GCM). The results are averaged over all the subjects (mean, thick line and SD, thin lines). No significant differences are observed as a function of the acceleration factor (blue: reference scan, red: 3.14X, orange: 4.09X, green: $4.89 \mathrm{X}$ and purple: $6.41 \mathrm{X}$ ) for the peak strain rates during contraction (frame \#9) and during relaxation (frame \#3).

FIGURE S3 time evolution of the three strain rate eigenvalues $\left(s_{-}, s_{+}\right.$, and $\left.s_{3}\right)$ for the Soleus muscle (SOL). The results are averaged over all the subjects (mean, thick line and SD, thin lines). No significant differences are observed as a function of the acceleration factor (blue: reference scan, red: $3.14 \mathrm{X}$, orange: $4.09 \mathrm{X}$, green: $4.89 \mathrm{X}$ and purple: $6.41 \mathrm{X}$ ) for the peak strain rates during contraction (frame \#9) and during relaxation (frame \#3).

FIGURE S4 time evolution of the three strain rate eigenvalues $\left(s_{-}, s_{+}\right.$, and $\left.s_{3}\right)$ for the Tibialis Anterior muscle (TA). The results are averaged over all the subjects (mean, thick line and SD, thin lines). No significant differences are observed as a function of the acceleration factor (blue: reference scan, red: 3.14X, orange: 4.09X, green: $4.89 \mathrm{X}$ and purple: $6.41 \mathrm{X}$ ) for the peak strain rates during contraction (frame \#3) and during relaxation (frame \#9).

How to cite this article: Mazzoli V, Gottwald LM, Peper ES, et al. Accelerated 4D phase contrast MRI in skeletal muscle contraction. Magn Reson Med. 2018;80:1799-1811. https://doi.org/10.1002/mrm. 27158 